\title{
Un problema de Dirichlet del tipo $p(x)$-Kirchhoff vía alternativa de Fredholm
}

Willy David Barahona Martíneł $\}^{1}$, Jesús Virgilio Luque Rivera ${ }^{2}$, José Simeón Quique Broncan $\oint^{3}$, Luis Enrique Quispe Gallegos ${ }^{4}$, Rocío Julieta De La Cruz Marcacuzcd ${ }^{5}$ y Edinson Raúl Montoro Alegre 6

Resumen: El objetivo de este trabajo es obtener soluciones débiles para una clase de problema de tipo $p(x)$ - Kirchhoff. El resultado es obtenido mediante teoremas del tipo Fredholm para dos operadores no lineales. Además, se considera la unicidad de las soluciones débiles.

Palabras claves: Ecuaciones de tipo $p(x)$-Kirchhoff, Resultados tipo Fredholm, Condición límite sin flujo.

\section{A Dirichlet problem of type $p(x)$ - Kirchhoff via Fredholm alternative}

\begin{abstract}
The objective of this work is to obtain weak solutions for a type of problem of type $p(x)$ - Kirchhoff. The result is obtained by Fredholm-type results for a pair of non-linear operators. In addition, the uniqueness of weak solutions is considered.
\end{abstract}

Keywords: $p(x)$-Kirchhoff type equations, Fredholm-type results, No-flux boundary condition.

Recibido: 19/05/2021. Aceptado: 12/06/2021. Publicado online: 18/06/2021.

(c) Los autores. Este artículo es publicado por la Revista PESQUIMAT de la Facultad de Ciencias Matemáticas, Universidad Nacional Mayor de San Marcos. Este es un artículo de acceso abierto, distribuido bajo los términos de la licencia Creative Commons Atribucion-No Comercia-CompartirIgual 4.0 Internacional.(http://creativecommons.org/licenses/by-nc-sa/4.0/) que permite el uso no comercial, distribución y reproducción en cualquier medio, siempre que la obra original sea debidamente citada. Para uso comercial, por favor póngase en contacto y reproduccion en cualquier medio, siempre que 1 con revistapesquimat.matematica@unmsm.edu.pe
con

\footnotetext{
${ }^{1}$ UNMSM, Facultad de Ciencias Matemáticas. e-mail: wbarahonam@unmsm.edu.pe

${ }^{2}$ UNMSM, Facultad de Ciencias Matemáticas, e-mail: jluquer@unmsm.edu.pe

${ }^{3}$ UNMSM, Facultad de Ciencias Matemáticas, e-mail: jquiqueb@unmsm.edu.pe

${ }^{4}$ UNMSM, Facultad de Ciencias Matemáticas, e-mail:luisenrique.quispe@unmsm.edu.pe

${ }^{5}$ UNMSM, Facultad de Ciencias Matemáticas, e-mail: rdelacruzm@unmsm.edu.pe

${ }^{6}$ UNMSM, Facultad de Ciencias Matemáticas, e-mail: emontoroa@unmsm.edu.pe
} 


\section{Introducción}

En el presente trabajo, tenemos como objeto de estudio, el siguiente problema $p(x)$-Kirchhoff

$$
\begin{aligned}
-M\left(\int_{\Omega} A(x, \nabla u)\right) \operatorname{div}(a(x, \nabla u)) & =f(x, u)|u|_{s(x)}^{t(x)} \text { en } \quad \Omega \\
u & =0 \quad \text { sobre } \partial \Omega
\end{aligned}
$$

donde $\Omega$ es un dominio acotado de $\mathbb{R}^{n}$ con frontera regular $\partial \Omega$, y $n \geq 1, p, s, t \in C(\bar{\Omega})$ para cada $x \in \bar{\Omega} ; M: \mathbb{R}^{+} \rightarrow \mathbb{R}^{+}$es una función continua, $f$ es una función de Caratheodory y $\operatorname{div}(a(x, \nabla u))$ es un operador del tipo $p(x)$-Laplaciano.

En los últimos años los estudios de ecuaciones diferenciales y problemas variacionales con condiciones de crecimiento no estándar han recibido considerable atención (Ver[2] - [4]); esto debido a las multiples aplicaciones a la Dinámica poblacional, Biomatemática, Mecánica elástica, Restauración de imágenes entre otras.

Consideramos (1) y estudiaremos la existencia, unicidad y singularidad de las soluciones débiles; para lograr lo deseado utilizaremos resultados topológicos del tipo Fredholm dados por Dinca en [1].

\section{Preliminares}

Mencionamos algunas propiedades y resultados básicos del espacio de Lebesgue generalizado $L^{p(x)}(\Omega)$ y del espacio de Sobolev generalizado $W_{0}^{1, p(x)}(\Omega)$; consideramos el conjunto:

$$
\begin{aligned}
& C_{+}(\bar{\Omega})=\{p(x) \in C(\bar{\Omega}): p(x)>1, \forall x \in \bar{\Omega}\} \\
p^{+}= & \max \{p(x) ; x \in C(\bar{\Omega})\} \text { y para algún } p(x) \in C_{+}(\bar{\Omega}) \\
p^{-}= & \min \{p(x) ; x \in C(\bar{\Omega})\} \text { y para algún } p(x) \in C_{+}(\bar{\Omega})
\end{aligned}
$$

Sea $M(\Omega)$ es el conjunto de todas las funciones reales medibles definidas sobre $\Omega$.

i,e) $M(\Omega)=\{u: u$ es una función medible con valor real sobre $\Omega\}$.

Definimos el Espacio de Lebesgue con exponente variable

$$
L^{p(x)}(\Omega)=\left\{u \in M(\Omega): \int_{\Omega}|u(x)|^{p(x)} d x<\infty\right\}
$$

con la norma

$$
|u|_{p(x)}=\inf \left\{\mu>0: \int_{\Omega}\left|\frac{u(x)}{\mu}\right|^{p(x)} d x \leq 1\right\}
$$

así $\left(L^{p(x)}(\Omega),|u|_{p(x)}\right)$ es un Espacio de Banach. 
Definimos el Espacio de Sobolev con exponente variable

$$
W^{1, p(x)}(\Omega)=\left\{u \in L^{p(x)}(\Omega):|\nabla u| \in L^{p(x)}(\Omega) .\right\}
$$

cuya norma está dada por

$$
\|u\|=|u|_{p(x)}+|\nabla u|_{p(x)}
$$

A la clausura de $C_{0}^{\infty}(\Omega)$ en $W^{1, p(x)}(\Omega)$ será denotada con $W_{0}^{1, p(x)}(\Omega)$. Se sabe que $|\nabla u|_{p(x)}$ y $\|u\|$ son normas equivalentes en $W_{0}^{1, p(x)}(\Omega)$. Por lo que usaremos la norma

$$
\|u\|=|\nabla u|_{p(x)}, \forall u \in W_{0}^{1, p(x)}(\Omega) .
$$

Proposición 2.1 Los espacio $L^{p(x)}(\Omega)$ y $L^{p^{\prime}(x)}(\Omega)$ son conjugados, donde $\frac{1}{p(x)}+\frac{1}{p^{\prime}(x)}=1$. Donde para cada $u \in L^{p(x)}(\Omega)$ y $v \in L^{p^{\prime}(x)}(\Omega)$ se cumple una desigualdad del tipo Hölder.

$$
\left|\int_{\Omega} u v d x\right| \leq\left(\frac{1}{p^{-}}+\frac{1}{\left(p^{-}\right)^{\prime}}\right)|u|_{p(x)}|v|_{p^{\prime}(x)}
$$

Demostración. Ver [7]pag. 20.

Proposición 2.2 Si $p^{-}>1$, entonces el espacio generalizado $L^{p(x)}(\Omega)$ es un espacio de Banach Separable y Reflexivo.

Demostración. Ver [7]pag. 17-21.

Proposición 2.3 Los espacios generalizados $W^{1, p(x)}(\Omega)$ y $W_{0}^{1, p(x)}(\Omega)$ son espacios de Banach Separables y Reflexivos.

Demostración. Ver [7]pag. 32-33.

Proposición 2.4 Denotamos $\rho(u)=\int_{\Omega}|u(x)|^{p(x)} d x . \forall u, u_{\nu} \in L^{p(x)}(\Omega)$, entonces tenemos las siguientes propiedades:

(1) $u \neq 0,|u|_{p(x)}=\lambda \Longleftrightarrow \rho\left(\frac{u}{\lambda}\right)=1$;

(2) $|u|_{p(x)}<1(=1 ;>1) \Longleftrightarrow \rho(u)<1(=1 ;>1)$;

(3) $S i|u|_{p(x)}>1, \Longrightarrow|u|_{p(x)}^{p^{-}} \leq \rho(u) \leq|u|_{p(x)}^{p^{+}}$;

(4) $S i|u|_{p(x)}<1, \Longrightarrow|u|_{p(x)}^{p^{+}} \leq \rho(u) \leq|u|_{p(x)}^{p^{-}}$;

(5) $\lim _{\nu \rightarrow+\infty}\left|u_{\nu}\right|_{p(x)}=0 \Longleftrightarrow \lim _{\nu \rightarrow+\infty} \rho\left(u_{\nu}\right)=0$;

(6) $\lim _{\nu \rightarrow+\infty}\left|u_{\nu}\right|_{p(x)}=+\infty \Longleftrightarrow \lim _{\nu \rightarrow+\infty} \rho\left(u_{\nu}\right)=+\infty$.

Demostración. Ver [7]pag. 16.

Proposición 2.5 (Desigualdad de Poincaré). Existe una constante $C_{0}>0$ talque

$$
|u|_{L^{p(x)}(\Omega)} \leq C_{0}|\nabla u|_{L^{p(x)}(\Omega)}, \quad \forall u \in W_{0}^{1, p(x)}(\Omega) .
$$


Demostración. Ver [7]pag. 37.

Proposición 2.6 Si $\mu \in C_{+}(\bar{\Omega})$ y $\mu(x) \leq p^{*}(x)\left(\mu(x)<p^{*}(x)\right)$ para $x \in \bar{\Omega}$, entonces existe una inmersión continua (compacta) $W_{0}^{1, p(x)}(\Omega) \hookrightarrow L^{\mu(x)}(\Omega)$, donde

$$
p^{*}(x)= \begin{cases}\frac{\left.n_{p(x}\right)}{n-p(x)}, & p(x)<n \\ +\infty, & p(x) \geq n .\end{cases}
$$

Demostración. Ver [7]pag. 37.

Definición 2.7 La función $u \in W_{0}^{1, p(x)}(\Omega)$ es llamada solución débil del problema (1) si

$$
-M\left(\int_{\Omega} A(x, \nabla u) d x\right) \int_{\Omega}(a(x, \nabla u)) \nabla v d x=|u|_{s(x)}^{t(x)} \int_{\Omega} f(x, u) v d x \quad \forall v \in W_{0}^{1, p(x)}(\Omega)
$$

En lo que continúa, la función $f: \Omega \times \mathbb{R} \longrightarrow \mathbb{R}$ siempre sera considerada con la condición de Caratheodory y además satisface:

$$
\left(f_{0}\right) \quad f(x, t) t \leq c_{1}|t|^{\alpha(x)-1}+c_{2}, \quad \forall(x, t) \in \Omega \times \mathbb{R}
$$

donde $c_{1}, c_{2}$ son constantes positivas, $\alpha \in C_{+}(\bar{\Omega})$ tal que

$$
1<\alpha(x)<p^{*}(x)
$$

$\left(M_{0}\right) \quad M:\left[0 ;+\infty\left[\longrightarrow\left[m_{0} ;+\infty\left[\right.\right.\right.\right.$ es continua no decreciente con $m_{0}>0$.

La aplicación

$$
a(x, \xi): \bar{\Omega} \times \mathbb{R}^{n} \longrightarrow \mathbb{R}
$$

es la derivada continua respecto a la variable $\xi$ de la aplicación

$$
A: \bar{\Omega} \times \mathbb{R}^{n} \longrightarrow \mathbb{R}^{n}, A=A(x, \xi) ;
$$

i,e) $a(x, \xi)=D_{\xi} A(x, \xi)=\frac{\partial}{\partial \xi} A(x, \xi)$, que satisface las siguientes condiciones:

(a) $a(x, \xi) \leq c_{0}\left(1+|\xi|^{p(x)-1}\right), \forall x \in \Omega$ y $\xi \in \mathbb{R}^{n}$ para alguna constante $c_{0}>0$.

(b) A es $p(x)$-uniformemente convexa, si existe una constante $k>0$ tal que

$$
A\left(x, \frac{\xi+\psi}{2}\right) \leq \frac{1}{2} A(x, \xi)+\frac{1}{2} A(x, \psi)-k|\xi-\psi|^{p(x)}, \forall x \in \Omega ; \xi, \psi \in \mathbb{R}^{n} .
$$

(c) $a(x, \xi) . \xi \leq p(x) A(x, \xi)$ para todo $x \in \bar{\Omega} ; \xi \in \mathbb{R}^{n}$.

(d) $A(x, 0)=0, \forall x \in \bar{\Omega}$.

(e) $(a(x, \xi)-a(x, \eta)) \cdot(\xi-\eta) \geq \gamma|\xi-\eta|^{p(x)}$, para algún $\gamma \geq 1$.

Observamos que el problema (1) no es variacional, por lo que nuestra herramienta en la búsqueda de soluciones es un resultado del tipo alternativa de Fredholm para un par de operadores no lineales obtenidos por G. Dinca [1].

Denotamos al operador grado de Leray-Schauder como $d_{L S}(G, B, 0)$. 
Teorema 2.8 (Dinca) Sean $X$ e $Y$ espacios de Banach reales y dos operadores no lineales $T, S: X \rightarrow Y$ tal que:

1. $T$ es biyectiva $y T^{-1}$ es continua.

2. $S$ es compacto.

3. Sea $\lambda \neq 0$ un número real talque: $\|(\lambda T-S)(x)\| \rightarrow+\infty$ cuando $\|x\| \rightarrow+\infty$

4. Existe una constante $R>0$ tal que

$$
\|(\lambda T-S)(x)\|>0 \text { si }\|x\| \geq R, \quad d_{L S}\left(I-T^{-1}\left(\frac{S}{\lambda}\right), B(\theta, R), 0\right) \neq 0
$$

\section{Entonces}

$\lambda I-S$ es suryectiva de $X$ en $Y$.

Demostración. Ver [1].

Recordemos que $u \in W_{0}^{1, p(x)}(\Omega)$ es una solución débil del problema (1) sí y sólo sí

$$
-M\left(\int_{\Omega} A(x, \nabla u)\right) \operatorname{div}(a(x, \nabla u))=|u|_{s(x)}^{t(x)} N_{f} u \quad \text { en } \quad W^{-1, p^{\prime}(x)}(\Omega)
$$

donde $N_{f}$ es el operador de Nemytski asociado a $f$.

\section{Resultado Principal}

Teorema 3.1 Si las siguientes condiciones se satisfacen:

(a) $a(x, \xi) \leq c_{0}\left(1+|\xi|^{p(x)-1}\right), \forall x \in \Omega$ y $\xi \in \mathbb{R}^{n}$ para alguna constante $c_{0}>0$. "

(b) A es $p(x)$-uniformemente convexo, si existe una constante $k>0$ tal que

$$
A\left(x, \frac{\xi+\psi}{2}\right) \leq \frac{1}{2} A(x, \xi)+\frac{1}{2} A(x, \psi)-k|\xi-\psi|^{p(x)}, \forall x \in \Omega ; \xi, \psi \in \mathbb{R}^{n} .
$$

(c) $a(x, \xi) \cdot \xi \leq p(x) A(x, \xi), \forall x \in \bar{\Omega} y \xi \in \mathbb{R}^{n}$.

(d) $A(x, 0)=0, \forall x \in \bar{\Omega}$.

(e) $(a(x, \xi)-a(x, \eta)) \cdot(\xi-\eta) \geq \gamma|\xi-\eta|^{p(x)}$, para algún $\gamma \geq 1$." y además

$$
f(x, t) t \leq c_{1}|t|^{\alpha(x)-1}+c_{2}, \quad \forall(x, t) \in \Omega \times \mathbb{R},
$$

$$
M:\left[0 ;+\infty\left[\longrightarrow \left[m_{0} ;+\infty\left[\text { es continua no decreciente con } m_{0}>0\right. \text {. }\right.\right.\right.
$$

$$
\frac{t^{+}+\alpha^{+}}{2}<p^{-}
$$

Entonces, existe una solución débil para el problema (1).

Prueba: Para aplicar el teorema 1, tomamos $Y=W^{-1, p^{\prime}(x)}(\Omega)$ y los operadores $T, S$ : $W_{0}^{1, p(x)}(\Omega) \rightarrow W^{-1, p^{\prime}(x)}(\Omega)$ de la siguiente manera

$$
\begin{gathered}
\langle T u, v\rangle=M\left(\int_{\Omega} A(x, \nabla u) d x\right) \int_{\Omega} a(x, \nabla u) \nabla v d x \\
\langle S u, v\rangle=|u|_{s(x)}^{t(x)}\left\langle N_{f} u, v\right\rangle
\end{gathered}
$$




$$
\langle S u, v\rangle=|u|_{s(x)}^{t(x)} \int_{\Omega} f(x, u) v d x
$$

Dado que $W_{0}^{1, p(x)}(\Omega) \hookrightarrow L^{\alpha(x)}(\Omega)$ es compacta, de la condición dada por $\left(f_{0}\right)$, podemos ver que la aplicación

$$
N_{f}: W_{0}^{1, p(x)}(\Omega) \rightarrow W^{-1, p^{\prime}(x)}(\Omega)
$$

es secuencialmente débilmente y fuertemente continua; la continuidad de $S$ es imediata.

Sea :

$$
\left(u_{\nu}\right)_{\nu \geq 1} \subseteq W_{0}^{1, p(x)}(\Omega) \quad \operatorname{acotad} a,
$$

entonces existe una subsucesión

$$
\left(u_{\nu_{k}}\right)_{k \geq 1} \quad \text { de } \quad\left(u_{\nu}\right)_{\nu \geq 1}
$$

tal que

$$
\left(N_{f}\left(u_{\nu_{k}}\right)\right)_{k \geq 1} \text { es fuertemente convergente }
$$

Sea:

$$
u_{\nu} \rightarrow u \quad \text { en } \quad W_{0}^{1, p(x)}(\Omega)
$$

entonces

$$
u_{\nu} \rightarrow u \quad \text { en } \quad L^{s(x)}(\Omega) \quad y \quad L^{\alpha(x)}(\Omega)
$$

de donde

$$
u_{\nu} \rightarrow u \quad \text { ctp } \quad \text { en } \quad \Omega
$$

Desde que $t \in C(\bar{\Omega})$, tenemos

$$
\left|u_{\nu}\right|_{s(x)}^{t(x)} \rightarrow|u|_{s(x)}^{t(x)} \quad \text { ctp } \quad x \in \Omega
$$

además

$$
f\left(x, u_{\nu}\right) \rightarrow f(x, u) \quad \text { ctp } \quad x \in \Omega
$$

entonces tenemos

$$
f\left(x, u_{\nu}\right)\left|u_{\nu}\right|_{s(x)}^{t(x)} \rightarrow f(x, u)|u|_{s(x)}^{t(x)} \quad \text { ctp } \quad x \in \Omega
$$

de $\left(f_{0}\right)$ y $u_{\nu} \rightarrow u \quad c t p \quad$ en $\Omega$

$$
\left.\left|f\left(x, u_{\nu}\right)\right| u_{\nu}\right|_{s(x)} ^{t(x)}-\left.f(x, u)|u|_{s(x)}^{t(x)}\right|^{\alpha^{\prime}(x)} \leq C\left(1+k(x)+|u|^{\alpha(x)}\right)
$$

donde $\left(1+k(x)+|u|^{\alpha(x)}\right) \in L^{1}(\Omega)$ y por el teorema de la Convergencia Dominada

$$
\left.\lim _{\nu \rightarrow+\infty}\left|\int_{\Omega}\right| u_{\nu}\right|_{s(x)} ^{t(x)} f\left(x, u_{\nu}\right)-\left.|u|_{s(x)}^{t(x)} f(x, u)\right|^{\alpha^{\prime}(x)} d x=0
$$

entonces

$$
\left.\lim _{\nu \rightarrow+\infty}|| u_{\nu}\right|_{s(x)} ^{t(x)} f\left(x, u_{\nu}\right)-\left.|u|_{s(x)}^{t(x)} f(x, u)\right|^{\alpha^{\prime}(x)}=0
$$

luego de (3) tenemos

$$
\left|\left\langle S u_{\nu}, v\right\rangle-\langle S u, v\rangle\right| \leq\left. C\left|f\left(x, u_{\nu}\right)\right| u_{\nu}\right|_{s(x)} ^{t(x)}-\left.f(x, u)|u|_{s(x)}^{t(x)}\right|_{\alpha^{\prime}(x)} \rightarrow 0
$$


Así, $S u_{\nu} \rightarrow S u$ en $W^{-1, p^{\prime}(x)}(\Omega)$. Esto demuestra que $S$ es compacto.

Por otro lado, consideramos la funcional $L: W_{0}^{1, p(x)}(\Omega) \rightarrow \mathbb{R}$ definida por

$$
L(u)=\int_{\Omega} A(x, \nabla u) d x, \forall u \in W_{0}^{1, p(x)}(\Omega)
$$

cuya derivada de Gateaux en el punto $u \in W_{0}^{1, p(x)}(\Omega)$ es dada por

$$
\left\langle L^{\prime}(u), v\right\rangle=\int_{\Omega} a(x, \nabla u) \nabla v d x, \forall u, v \in W_{0}^{1, p(x)}(\Omega)
$$

La monotonía estricta de $L^{\prime}$ es deducida inmediatamente de la condición de monotonicidad (a), de (e) y de la siguiente identidad elemental $\forall a, b \in \mathbb{R}^{n}$ se cumple:

$$
\begin{gathered}
2^{2-p}|a-b|^{p} \leq\left(|a|^{p-2} a-|b|^{p-2} b\right)(a-b) ; \quad \text { si } \quad p(x) \geq 2 \\
(p-1)|a-b|^{2}(|a|+|b|)^{p-2} \leq\left(|a|^{p-2} a-|b|^{p-2} b\right)(a-b) ; \quad \text { si } \quad 1<p(x)<2
\end{gathered}
$$

donde estamos usando el producto interno estandar de $\mathbb{R}^{n}$. Así tenemos que $L$ es estrictamente convexo.

Dado que $M$ es no decreciente, entonces $\widehat{M}(t)=\int_{0}^{t} M(s) d s$ es convexo en $[0,+\infty[$ así, para cada $u, v \in W_{0}^{1, p(x)}(\Omega)$, con $u \neq v$, y cada $\left.s, t \in\right] 0,1[$ con $s+t=1$ se tiene

$$
\widehat{M}(L(s u+t v))<\widehat{M}(s L(u)+t L(v)) \leq s \widehat{M}(L(u))+t \widehat{M}(L(v))
$$

Esto muestra que

$$
\phi(u)=\widehat{M}\left(\int_{\Omega} A(x, \nabla u)\right)
$$

es estrictamente convexo, y como

$$
\left\langle\phi^{\prime}(u), v\right\rangle=\langle T u, v\rangle
$$

deducimos que $T$ es estrictamente monótona en $W_{0}^{1, p(x)}(\Omega)$, por lo que $T$ es una inyección, lograndose probar que $T$ es un operador del tipo $\left(S_{+}\right)$.

Ahora, para $u \in W_{0}^{1, p(x)}(\Omega)$, con $\|u\|>1$, tenemos

$$
\frac{\langle T u, u\rangle}{\|u\|} \geq m_{0}\|u\|^{p-1} \longrightarrow+\infty
$$

cuando $\|u\| \rightarrow+\infty$ por lo tanto, $T$ es coercivo; los argumentos estándar garantizan que $T$ es demicontinuo.

Por el teorema de Minty-Browder, el operador $T$ es una suryección, y por lo tanto la inversa $(T)^{-1}: W^{-1, p^{\prime}(x)}(\Omega) \rightarrow W_{0}^{1, p(x)}(\Omega)$ de $T$ existe.

Ahora probaremos la continuidad de $(T)^{-1}$.

En efecto sean:

$$
\left(g_{\nu}\right)_{\nu \geq 1} \subseteq W^{-1, p^{\prime}(x)}(\Omega) \quad \text { talque } \quad g_{\nu} \rightarrow g \quad \text { en } \quad W^{-1, p^{\prime}(x)}(\Omega)
$$

$\mathrm{y}$

$$
u_{\nu}=(T)^{-1} g_{\nu}, \quad u=(T)^{-1} g, \quad \text { entonces } \quad T u_{\nu}=g_{\nu}, \quad T u=g
$$


Por la coercitividad de $T$, deducimos que $\left(u_{\nu}\right)_{\nu \geq 1}$ es acotada en $W_{0}^{1, p(x)}(\Omega)$. así tenemos que

$$
u_{\nu} \rightarrow u \quad \text { en } \quad W_{0}^{1, p(x)}(\Omega)
$$

desde que

$$
\begin{gathered}
g_{\nu} \rightarrow g \text { en } \quad W^{-1, p^{\prime}(x)}(\Omega) \\
\lim _{\nu \rightarrow+\infty}\left\langle T u_{\nu}-T u, u_{\nu}-u\right\rangle=\lim _{\nu \rightarrow+\infty}\left\langle g_{\nu}-g, u_{\nu}-u\right\rangle=0 .
\end{gathered}
$$

Como $T$ es del tipo $\left(S_{+}\right)$y $u_{\nu} \rightarrow u$ entonces $T^{-1}$ es continuo.

Por otro lado después de algunos cálculos obtenemos

$$
\begin{gathered}
\|T u\| \geq m_{0}\|u\|^{2 p^{-}-1}, \forall u \in W_{0}^{1, p(x)}(\Omega),\|u\| \geq 1, \\
\|S u\| \geq c_{1}\|u\|^{t^{+}+\alpha^{+}-1}+c_{2}, \forall u \in W_{0}^{1, p(x)}(\Omega),\|u\| \geq 1
\end{gathered}
$$

Por lo que para $\|u\| \geq 1$ tenemos:

$$
\|(T-S) u\| \geq\|T u\|-\|S u\| \geq m_{0}\|u\|^{2 p^{-}-1}-c_{1}\|u\|^{t^{+}+\alpha^{+}-1}+c_{2}, \forall u \in W_{0}^{1, p(x)}(\Omega)
$$

Luego concluimos que

$$
\|(T-S) u\| \rightarrow+\infty \quad \text { cuando } \quad\|u\| \rightarrow+\infty
$$

entonces existe $r_{1}>1$ tal que

$$
\|(T-S) u\|>1, \forall u \in W_{0}^{1, p(x)}(\Omega), \quad \text { con } \quad\|u\|>r_{1}
$$

luego realizando cálculos simples se demuestra que

$$
A=\left\{u \in W_{0}^{1, p(x)}(\Omega): u=t T^{-1}(S u) ; t \in[0,1]\right\}
$$

es acotado en $W_{0}^{1, p(x)}(\Omega)$

Entonces existe $r_{2}>0$ tal que $A \subseteq B\left(0, r_{2}\right)$.

Elegimos $R=\max \left\{r_{1}, r_{2}\right\}$ y consideramos la homotopía de las transformaciones compactas

$$
H_{1}:[0,1] \times \bar{B}(0, R) \longrightarrow W_{0}^{1, p(x)}(\Omega)
$$

definida por

$$
H_{1}(t, u)=t T^{-1}(S u)
$$

de la elección de $R$, tenemos $H_{1}(t, u) \neq u, \quad \forall u \in \partial \bar{B}(0, R)$, por lo tanto el grado de LeraySchauder

$$
d_{L S}\left(I-T^{-1}(S), B(0, R), 0\right)
$$

esta bien definido. También

$$
d_{L S}\left(I-T^{-1} \circ S, B(0, R), 0\right)=d_{L S}\left(I-H_{1}(1, .), B(0, R), 0\right) .
$$


Luego

$$
d_{L S}\left(I-T^{-1} \circ S, B(0, R), 0\right)=d_{L S}\left(I-H_{1}(0, .), B(0, R), 0\right)=d_{L S}(I, B(0, R), 0)=1 \neq 0
$$

Así, el par de operadores no lineales $(T, S)$ satisfacen la hipótesis del teorema (1) para $\lambda=1$.

Se deduce que

$$
T-S: W_{0}^{1, p(x)}(\Omega) \rightarrow W^{-1, p^{\prime}(x)}(\Omega)
$$

es suryectivo. Por lo tanto, existe $u \in W_{0}^{1, p(x)}(\Omega)$ tal que

$$
(T-S) u=0 ; \quad \text { en } \quad W^{-1, p^{\prime}(x)}(\Omega)
$$

es decir

$$
T u=S u \quad \text { en } \quad W^{-1, p^{\prime}(x)}(\Omega)
$$

de donde concluimos la demostración.

\section{Resultados}

Los resultados presentados aqui nos muestran la existencia de soluciones débiles para una clase de problema de tipo $p(x)$ - Kirchhoff, este resultado fue obtenido para dos operadores no lineales, utilizando el teorema de Minty-Browder, la homotopía de las transformaciones compactas y la teoría de grado de Leray-Schauder. Probando la unicidad de las soluciones débiles.

\section{Discusión}

Debido a que el problema (1) no es variacional, mostramos una herramienta para la búsqueda de soluciones, la cual está basada en un resultado del tipo alternativa de Fredholm para un par de operadores no lineales obtenidos por G. Dinca [1]. A partir de ello se obtiene el resultado buscado.

Una pregunta natural es ¿la técnica aplicada, seguira siendo válida en problemas con operadores fraccionales y con no linealidades que involucren términos gradientes? , ¿se obtendrá un mejor resultado?

\section{Conclusión}

1. Al no tener el problema (1)una estructura variacional, hemos recurrido a una técnica topológica dada por Dinca [1] . Esta metodología de trabajo nos ha permitido obtener solución débil del problema.

2. El método introducido es bastante general y es aplicable a ecuaciones elípticas con otros operadores y términos no lineales y en otros tipos de espacios, por ejemplo en los espacios de Orlicz.

3. Similarmente es posible aplicar el método a ecuaciones elípticas que presentan condiciones de frontera tipo Neumann, condiciones mixtas, de ningún flujo, etc. 


\section{Referencias bibliográficas}

[1] Dinca G.( 2001) A Fredholm-type result for a couple of nonlinear operators, CRACSI, $333(5),(2001), 415-419$.

[2] Dai G, Liu D.(2009) Infinitely many positive solutions for a $p(x)$-Kirchhoff type equation, J. Math. Anal Appl. 359(2009)704-710.

[3] Dai G, Hao R.(2009) Existence of solutions for a $p(x)$-Kirchhoff type equation, J. Math. Anal Appl. 359(2009)275-284.

[4] Fan X. L.(2010) On nonlocal $p(x)$-Laplacian Dirichlet problems. Nonlinear Anal. 72(2010)3314-3323.

[5] Barahona W, Cabanillas E, Rodriguez G, De La Cruz R, Quique J.(2014) Existence of solutions for a class of $p(x)$-Kirchhoff type equation via topological methods, Libro de Resumenes VIII ENAMA. UFPE-Pernambuco/ Brasil(2014)200-201.

[6] Correa, F.J.S.A., Costa, A.C.R., Figueiredo G.M.(2007) On a singular elliptic problem involving the $p(x)$ - Laplacian and generalized Lebesgue - Sobolev spaces. Advances in Mathematical Sciences and Applications, Vol. 17.

[7] Guimaraes, C.J.(2006) Sobre os Espacos de Lebesgue e Sobolev Generalizados e Aplicacoes Envolvendo o p (x)-Laplaciano. Dissertacao de mestrado, CCT - UFCG.

[8] J.L. Lions.(1978) On some questions in boundary value problems of mathematical physics . Instituto de Matematica, UFRJ, Rio de Janeiro, RJ.

[9] Medeiros, L.A. \& Milla, M.A.(2000) Espaços de Sobolev(Iniciação aos Problemas Elípticos não Homogêneos) . IM-UFRJ, Rio de Janeiro.

[10] Hästö, P.A.(2005) The $p(x)$ - Laplacian and applications, National Conference on PDE and Applications . Coimbatore, Índia.

[11] Adams, R. (1975). Sobolev Spaces. United States of America, New York: Academic Press.

[12] Brezis, H. (1983). Analyse Fonctionnelle - Théorie et Applications. Francia, Paris: Mason.

[13] Kesavan, S. (1990). Topics in Functional Analysis and Applications. India, New Delhi: Jhon Willey \& Sons.

[14] Lee, R. y Carter, L. (1992). Modeling and Forecasting U.S. Mortality. Journal of the American Statistical Association, 87 3(1), 659-671.

[15] Tineo, M. (2017). Existencia de soluciones para una clase de sistemas elípticos semilineales (tesis de maestría en Matemática Pura). Universidad Nacional Mayor de San Marcos, Lima, Perú. 